\title{
A group-administered test of children's metamemory
}

\author{
JOHN M. BELMONT \\ University of Kansas Medical Center, Kansas City, Kansas \\ and \\ JOHN G. BORKOWSKI \\ University of Notre Dame, Notre Dame, Indiana
}

\begin{abstract}
A group-administered test of children's metamemory was assessed for retest reliability, internal consistency, sensitivity to age, and sensitivity to IQ. In all important psychometric respects, this group test was similar to previously published individually administered tests. The new test provides an easily administered, broad-based index of memory-related knowledge for young children.
\end{abstract}

Flavell and Wellman's (1977) theoretical treatment of metamemory has inspired many research programs to seek a satisfactory empirical base for the concept (see Cavanaugh \& Perlmutter, 1982, for a review). The early studies were theoretically rather shallow, leading some commentators to question the construct validity of metamemory itself (Cavanaugh \& Perlmutter, 1982; Wellman, 1983). In contrast, recent research has adduced more precise theoretical formulations (e.g., searching for metamemory-memory relationships on difficult tasks or on transfer tests that demand deliberate, active processing); clearer definitions of metamemory; and a longitudinal, multidimensional perspective on how metamemory operates in interaction with other dispositional and cognitive variables to determine the acquisition of new, complex memory strategies (Borkowski, Reid, \& Kurtz, 1984; Campione \& Brown, 1984).

Considering the poor reliability of many metamemory measures (Cavanaugh \& Perlmutter, 1982), Schneider's (1985) meta-analysis of the entire literature showed a surprisingly strong overall relationship between metamemory and memory behavior (average $r=.42$ ). That value is sufficient, we feel, to justify continued development of metamemory tests. To date, metamemory research has relied on one-on-one tests that are time-consuming to administer and may not be better psychometrically or have

This research was supported by NIH Grants HD-15854 (to J. Belmont), HD-21218 (to J. Borkowski), and HD-02528 (to the Ralph L. Smith Research Center). We gratefully acknowledge the assistance of Martha Carr, Wayne Mitchell, Anna Hefferman, and Judy Coppaken in the collection and analysis of the data. We are indebted to Judy Burgess, Connie Stillwell, Connie Thompson, Jim Fletcher, Lois Roth, Jack Liles, and Wayne Morris (principal) of Highlands Elementary School, Fairway, Kansas, for their generous assistance and flexibility in permitting this study to go forward. Reprints and the metamemory test booklet and instructions can be obtained from John Belmont, Department of Pediatrics, University of Kansas Medical Center, Kansas City, KS 66103. higher construct validity than a group test. Thus, we developed a brief (30-min) group-administered metamemory test that yields a broad-based picture of a child's memoryrelated information and is probably applicable to children with mental ages of approximately 6 to 12 years. The group test was founded on portions of a one-on-one metamemory battery (Borkowski, Ryan, Kurtz, \& Reid, 1983; Kurtz, Reid, Borkowski, \& Cavanaugh, 1982) whose subtests ranged from memory estimation to knowledge about study strategies to ways to retrieve old facts.

\section{METHOD}

\section{Subjects}

There were 24 third graders and 20 fifth graders selected randomly from two Midwestern schools. Cognitive Abilities Test (CAT) scores were available for the fifth graders, all of whom had been given the CAT in the third grade.

\section{Materials and Procedures}

The metamemory battery (MMB) consisted of an $81 \frac{1}{2} \times 11$ in. booklet with 17 pages of five alternating colors that permitted proctors to easily monitor the 30-min group administration. The booklet comprised five subtests: Organized List, Preparation Object, Memory Estimation, Study Time for Paired Associates, and Study Time for Circular Recall. The first three of these were taken nearly intact from the Kurtz et al. (1982) test. The last two were developed de novo to provide checks on knowledge of the specific laboratory memory tasks used in an ongoing laboratory study of memory strategy development. None of the present subjects was involved in the laboratory work. The MMB was given twice (6-week interval) in the classroom to each grade separately. Specific items were as follows:

Organized List (LIST). Three pages each contained two lists of words to assess the children's understanding that categorical organization facilitates learning. For example, on one page one list included only fruits and vegetables, whereas the other list was completely heterogeneous with respect to category. The children were required to mark a box above one of the lists in response to the question, "Which list would be easier for you to remember?" Two points were given for each correct choice (maximum $=6$ points).

Preparation Object (SKATES). SKATES assessed the children's flexibility in thinking of ways to remember. They were asked to write 
down as many as seven different methods they might use some evening to remember to take a pair of skates to school the following morning. Responses were scored according to seven classes of method: (1) make written or pictorial reminders, (2) have somebody else remind them, (3) strategically place the skates, (4) rehearse or use some other in-thehead method, (5) set up an external reminder, (6) attach a reminder to oneself, and (7) seek additional methods. One point was allotted for each response class, and an extra point was given for a second instance of each class. An additional point was also awarded if more than three response classes were represented (maximum $=8$ points).

Memory Estimation (ME). This test assessed the ability to judge memory capacity. The children first predicted how many of 15 unrelated common words they could freely recall if they were given $1 \mathrm{~min}$ to study them. After the initial prediction $\left(\mathbf{P}_{1}\right)$, they were actually given $1 \mathrm{~min}$ to memorize a second set of 15 words and then had $1 \mathrm{~min}$ to write them down from memory. Finally, a third list of 15 words was presented, and the children made their second prediction $\left(\mathbf{P}_{2}\right)$ about how many they could recall if given $1 \mathrm{~min}$ of study time. The first prediction was scored as $4\left(1-\left|\mathrm{P}_{1}-A\right| / A\right)$, where $A$ was the actual number recalled from the second list. The second prediction was scored $5\left(1-\left|\mathrm{P}_{2}-A\right| / A\right)$. Negative scores were recorded as zeros. The second formula weighted a hypothesized increase in awareness of memory capacities produced by the practice trial. The two scores were summed to obtain overall $\mathrm{ME}$ score (maximum $=9$ points).

Study Time for Paired Associates (PA). The PA test assessed the children's understanding of the appropriate study-time distribution for memorizing pairs of words. The task required the children to recognize the association values linking word pairs. If high interword associations existed, the appropriate strategy would be to devote relatively little time to memorizing those pairs and to allot more time to pairs having low interword associations. Four pairs of words were presented, including two pairs of high interword associations (e.g., circle-square) and two of low interword associations (e.g., doll-tree). The word pairs were randomly listed. The children were given a choice of three patterns of study time: (1) allot equal time to each of the four pairs (E, equal time), (2) allot less time to the related and more time to the unrelated pairs (C, the correct answer), (3) allot more time to the related and less time to the unrelated pairs (W, the wrong answer). Two pages, each with different pairs, were given to check for consistency of response choices; scoring depended on the response pattern. Children who chose equal time (E) or the correct answer (C) on the first trial yet chose the wrong answer (W) for the second trial (i.e., E,W or C,W) were awarded no points. We assumed in this case that the correct or equal answer was based on guessing rather than on strategic knowledge. The combinations of correct-correct $(\mathrm{C}, \mathrm{C})$, wrong-wrong $(\mathrm{W}, \mathrm{W})$, or equal-equal $(\mathrm{E}, \mathrm{E})$ were awarded the sum of the individual points: 6 points, 0 points, and 2 points, respectively. The combinations of equal-correct $(E, C)$ and wrong-correct $(\mathrm{W}, \mathrm{C})$ were awarded 4 and 2 points, respectively. In these instances, the child may have acquired a study strategy from the first trial and applied this skill to the second trial. Finally, the combination of correct-equal (C,E) was awarded 1 point; the assumption was that the child had some strategic knowledge but was not aware of the specific strategies needed for the task. This scoring procedure allowed for a sensitive assessment of children's strategic knowledge by taking into account pattern consistency in combination with differing levels of strategic knowledge as well as the possibility for strategy learning during the test (maximum $=6$ points).

Study Time for Circular Recall (CR). The CR test assessed the children's abilities to think through an appropriate allotment of study time for a string of six words in order to facilitate circular recall. The first hypothetical task required the child to recall in $3 / 3$ circular order (4-56-1-2-3) words that were presented serially from 1 to 6 . A multiplechoice test offered three possible strategy selections: (1) equal study time for all words (E), (2) more time for words 1-3 and less for words 4-6 (C), and (3) more time for words 4-6 than for words 1-3 (W). A second trial presented the hypothetical $2 / 4$ circular order (5-6-1-2-3-4) with response options similar to the $3 / 3$ task. Scoring was identical to that used for PA (maximum $=6$ points).

\section{RESULTS AND DISCUSSION}

Table 1 contains the subtest means for the two administrations of the MMB. As can be seen in the table, fifth graders were higher in memory knowledge than third graders on every subtest. These developmental differences are prerequisite for establishing the psychometric integrity of the metamemory battery.

Prior to conducting correlational analyses, the scores for each subject were transformed to $z$ scores within each subtest for each age group separately. This removed the age effect in all analyses involving the combined groups. The reliability of the composite metamemory score (summed across subtests) was assessed through a series of test-retest correlations: for third graders, $r(22)=.69$, $p<.001$; for fifth graders, $r(18)=.61, p<.01$; and for the two grades combined, $r(42)=.66, p<.001$. Within each grade, significant changes in composite metamemory scores from test to retest were also noted: for third graders, $t(23)=3.70, p<.01$; for fifth graders, $t(19)=2.81, p<.02$. In both cases the scores improved.

As for internal consistency, the sum of the SKATES and PA subtests correlated only marginally with the sum of the LIST, CR, and ME subtests $[r(42)=.23]$. In addition, most of the subtests were only weakly related to the sum of the remaining four subtests. The exception was PA, which correlated significantly with the sum of the other four subtests $[r(42)=.39, p<.02]$. The next highest intertest correlation was for ME, which approached significance when correlated with the sum of the four subtests $[r(42)=.21]$.

Consider how these findings compare with the corresponding results from the individually administered metamemory battery: (1) The test-retest reliabilities were highly significant and similar at .66 for the group test versus .67 for the individually administered test (Kurtz et al., 1982). (2) Internal consistency was not striking in the group test (only one of the five individual subtests correlated significantly with the sum of the remaining subtests). These results are consistent with those of Borkowski

Table 1

Mean First and Second MMB Item Scores for 3rd and 5th Graders

\begin{tabular}{|c|c|c|c|c|c|c|c|c|c|c|c|c|c|}
\hline \multirow[b]{2}{*}{ Grade } & \multirow[b]{2}{*}{$N$} & \multicolumn{2}{|c|}{ LIST } & \multicolumn{2}{|c|}{ SKATES } & \multicolumn{2}{|c|}{ ME } & \multicolumn{2}{|c|}{ PA } & \multicolumn{2}{|c|}{$\mathrm{CR}$} & \multicolumn{2}{|c|}{ Total } \\
\hline & & $1 \mathrm{st}$ & 2nd & $1 \mathrm{st}$ & 2nd & $1 \mathrm{st}$ & 2nd & $1 \mathrm{st}$ & 2nd & $1 \mathrm{st}$ & 2nd & $1 \mathrm{st}$ & 2nd \\
\hline 3 & 24 & 2.4 & 3.1 & 3.0 & 2.8 & 4.1 & 5.3 & 3.0 & 3.8 & 1.9 & 2.4 & 14.4 & 17.4 \\
\hline 5 & 20 & 3.1 & 4.0 & 3.5 & 3.8 & 6.3 & 7.3 & 4.5 & 4.3 & 2.5 & 3.1 & 19.9 & 22.5 \\
\hline Combined & 44 & 2.7 & 3.5 & 3.2 & 3.3 & 5.1 & 6.2 & 3.7 & 4.0 & 2.2 & 2.7 & 16.9 & 19.7 \\
\hline
\end{tabular}

Note-LIST = Organized List Test, SKATES = Preparation Object Test, ME = Memory Estimation Test, $\mathrm{PA}=$ Study Time for Paired Associates Test, $\mathrm{CR}=$ Study Time for Circular Recall Test. 
et al. (1983), in whose study of third graders' responses to individually administered metamemory items, none of the intersubtest correlations was significant. There is evidently a major developmental shift in internal consistency: First graders show high consistency (Borkowski et al., 1983), second graders show moderate consistency (Kurtz et al., 1982), and third graders, as well as older children, show low consistency (Borkowski et al., 1983, and the present data). Is metamemory related to general intelligence? For the individually administered version, Kurtz et al. (1982) reported that second graders' composite MMB scores correlated .46 with the Wechsler Intelligence Scale for Children (WISC) vocabulary subtest, and Borkowski et al. (1983) found correlations between WISC vocabulary and metacognition of .64 for first graders and .54 for third graders. These figures agree nicely with the current results for fifth graders' group-administered composite MMB scores and CAT IQ $[r(18)=.65$, $p<.01]$.

In summary, the group metamemory test is sensitive to age, and the comparisons of the individual and group tests show nearly identical test-retest reliability, internal consistency, and correlations with IQ. It thus appears that the new group test is psychometrically adequate for research that calls for an estimate of children's overall knowledge about memory states and processes.

\section{REFERENCES}

Borkowski, J. G., ReID, M. K., \& KURTZ, B. E. (1984). Metacognition and retardation: Paradigmatic, theoretical, and applied perspectives. In P. H. Brooks, R. Sperber, \& C. McCauley (Eds.), Learning and cognition in the mentally retarded (pp. 55-75). Hillsdale, $\mathrm{NJ}$ : Erlbaum.

Borkowski, J. G., Ryan, E. B., KuRTZ, B. E., \& ReID, M. K. (1983). Metamemory and metalinguistic development: Correlates of children's intelligence and achievement. Bulletin of the Psychonomic Society, 21, 393-396.

CAmpione, J. C., \& Brown, A. L. (1984). Learning ability and transfer propensity as sources of individual differences in intelligence. In P. H. Brooks, R. Sperber, \& C. McCauley (Eds.), Learning and cognition in the mentally retarded (pp. 265-293). Hillsdale, NJ: Erlbaum.

Cavanaugh, J. C., \& Perlmutrer, M. (1982). Metamemory: A critical examination. Child Development, 53, 11-28.

Flavell, J. H., \& Wellman, H. M. (1977). Metamemory. In R. Kail \& J. Hagen (Eds.), Perspectives on the development of memory and cognition (pp. 3-33). Hillsdale, NJ: Erlbaum.

Kurtz, B. E., Reid, M. K., Borkowski, J. G., \& Cavanaugh, J. C. (1982). On the reliability and validity of children's metamemory. Bulletin of the Psychonomic Society, 19, 137-140.

SCHNEIDER, W. (1985). Developmental trends in the metamemorymemory behavior relationship: An integrative review. In D. L. Forrest-Pressley, G. E. MacKinnon, \& T. G. Waller (Eds.), Cognition, metacognition, and performance: Vol. 1. Theoretical perspectives (pp. 57-109). New York: Academic Press.

Wellman, H. (1983). Metamemory revisited. In M. T. H. Chi (Ed.), Trends in memory development (Vol. 2, pp. 31-51). Basel, Switzerland: Karger. 